\title{
Bilateral Sesamoid Feet Fracture - A Rare Association
}

\section{Márcio Luís Duarte ${ }^{1 *}$, Bruno Fernandes Barros Brehme de Abreu1, Lucas Ribeiro dos Santos ${ }^{2}$, Laura Burni Pereira Gomes ${ }^{3}$ and Marcelo de Queiroz Pereira da Silva ${ }^{1}$}

${ }^{1}$ Department of Radiology, Webimagem, Brazil

${ }^{2}$ Faculdade de Ciências Médicas de Santos, Brazil

${ }^{3}$ Hospital Felício Rocho, Brazil

*Corresponding Author: Márcio Luís Duarte, Department of Radiology,

Webimagem, Brazil.

DOI: $10.31080 /$ ASOR.2020.03.0147
Received: February 05, 2020

Published: February 18, 2020

(C) All rights are reserved by Márcio Luís

Duarte., et al.

\begin{abstract}
Sesamoid bones are partially or totally embedded in the substance of a corresponding tendon. Anatomically they are part of a gliding mechanism that reduces friction and protects the tendon. The sesamoid complex consists of seven muscles, eight ligaments, and two sesamoid bones and it's pathological conditions include traumatic, degenerative, inflammatory, infectious, and ischemic processes. Most painful conditions involving the hallux sesamoid bones are, however, related to acute trauma or chronic stress. Medial tibial sesamoid bone fractures are rare and are often missed as they are treated as soft tissue injuries [4]. Bilateral fractures are extremely rare.
\end{abstract}

Keywords: Fractures; Bone/Diagnostic Imaging; Sesamoid Bones/Diagnostic Imaging; Magnetic Resonance Imaging

\section{Introduction}

Sesamoid bones are partially or totally embedded in the substance of a corresponding tendon [1]. Anatomically they are part of a gliding mechanism that reduces friction and protects the tendon $[1]$.

The medial and lateral hallux sesamoids are embedded within the medial and lateral slips of the flexor hallucis brevis tendon at the level of the first metatarsal head [1]. The purpose of the hallux sesamoid bones is to provide a mechanical advantage during hallux flexion, by reducing friction and reinforcing adjacent soft tissues [1]. The great toe complex transmits more than $50 \%$ of the bodyweight on exercise [2]. The size and shape of the hallux sesamoids vary considerably [1].

Case presentation

39 years-old man referring pain in the plantar region of both feet for 03 months. He refers to practice running for about 7 to 10 kilometers daily on the running belt for two years without using specific sneakers for such sport. Refers pain in palpation in the region of the first metatarsal head in both feet. Deny surgeries, diseases and the practice of other sports. Magnetic resonance imaging
(MRI) demonstrated a fracture of the medial sesamoid with slight edema in both feet (Figures 1 and 2). The patient stopped running for three months and refers no pain, but he didn't run again yet.

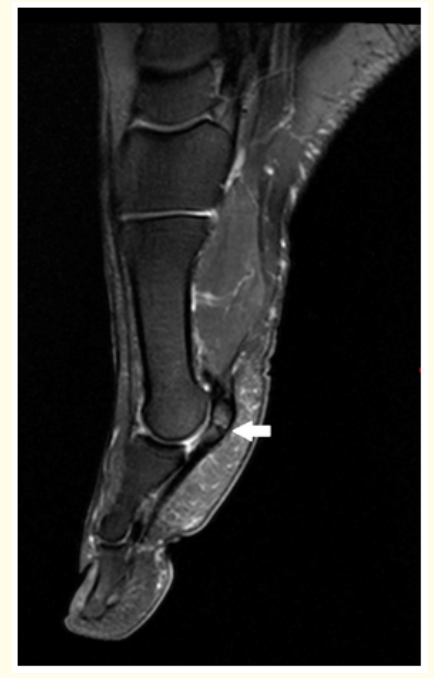

Figure 1: Right foot sagittal T2 FAT SAT MRI demonstrating fracture of the medial sesamoid with slight edema (white arrow). 


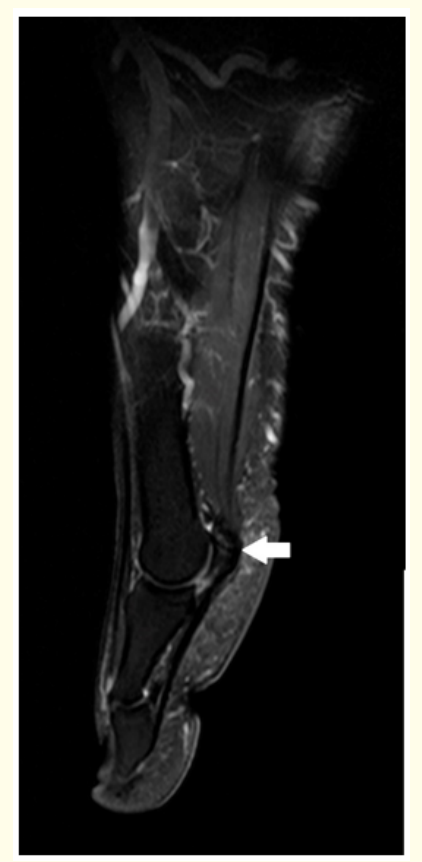

Figure 2: Left foot sagittal T2 FAT SAT MRI demonstrating fracture of the medial sesamoid with slight edema (white arrow).

\section{Discussion}

The sesamoid complex consists of seven muscles, eight ligaments, and two sesamoid bones and it's pathological conditions include traumatic, degenerative, inflammatory, infectious, and ischemic processes [1-3]. Most painful conditions involving the hallux sesamoid bones are, however, related to acute trauma or chronic stress [1].

Medial tibial sesamoid bone fractures are rare and are often missed as they are treated as soft tissue injuries [4]. Bilateral fractures are extremely rare [5]. The mechanism of injury in most of the cases is forced hyperextension of the great toe [4] that can result from direct trauma or a crush injury [5]. Chronic stress may also cause painful conditions in the hallux sesamoids, and is most commonly associated with chondromalacia, osteochondritis osteonecrosis and stress fracture [1].

Depending on the kind of sport, two etiologies are seen [6]. In soccer, football, or martial arts, for example, a traumatic sesamoid fracture from hyperextension of the great toe produces the acute onset of symptoms [6]. In endurance sports, such as running or dancing, chronic stress fractures of the sesamoid bone develop with an insidious onset of symptoms $[3,6,7]$. Approximately $1 \%$ of all running injuries involve the sesamoids [8].

The diagnosis of a sesamoid stress fracture is suggested by clinical symptoms [4]. Plain films are commonly normal [7]. From the radiological standpoint, distinguishing between bipartite sesamoid and true fracture of a tibial hallux sesamoid can be particularly challenging, and several suggestions have been made as to how to do so efficiently [1]. The single medial sesamoid with a fracture is slightly larger than the lateral sesamoid, whereas the bipartite sesamoid is much larger [1]. In addition, a fracture tends to show a sharp, radiolucent, uncorticated line, whereas the bipartite sesamoid has two corticated fragments [1]. The two fragments in a fractured sesamoid often fit together well, whereas the two components of a bipartite sesamoid do not [1].

In some cases, technetium bone scans and MR imaging may be used for improving diagnostic accuracy [1]. A 99mTc-MDP bone scan should be normal in a bipartite hallux but will show increased uptake in a fractured bipartite [1,9]. The MR imaging may also show bone marrow edema in a recently fractured sesamoid [1].

The computed tomography (CT scan), in sagittal and axial planes, was found to be the most reliable imaging method to discern a stress fracture in a painful fragmented sesamoid [9]. The most specific characteristic of the stress fracture is the broken cortical line with the separation of the fragments [9].

The treatment of the acute fractures is usually non-weight bearing in a cast with a toe plate for 6 weeks followed by walker boot for 4 to 6 more weeks [5]. Open reduction and internal fixation of acute fractures are controversial and typically reserved for widely displaced fractures [5]. Old fractures do not heal well even with extensive immobilization will demand further treatment, which consists of total sesamoid excision with the reconstruction of the flexor hallucis brevis tendon $[6,10,11]$.

\section{Acknowledgement}

Not applicable.

\section{Conflict of Interest}

The authors declare that there is no conflict of interests regarding the publication of this paper.

\section{Patient Consent}

The written informed consent of the patient was obtained, for the publication of her case.

\section{Bibliography}

1. Mellado JM., et al. "Accessory ossicles and sesamoid bones of the ankle and foot: imaging findings, clinical significance and differential diagnosis". European Radiology 13.6 (2003): L164L177.

2. Blundell CM., et al. "Percutaneous screw fixation for fractures of the sesamoid bones of the hallux". The Journal of Bone and Joint Surgery, British 84.8 (2002):1138-1141. 
3. Lee DK., et al. "Hallux, sesamoid, and first metatarsal injuries". Clinics in Podiatric Medicine and Surgery 28.1 (2011): 43-56.

4. Daddimani RM., et al. "Fracture of the medial tibial sesamoid bone of the foot-case report". Journal of Clinical and Diagnostic Research 9.4 (2015): RD03-RD04.

5. Cohen BE. "Hallux sesamoid disorders". Foot and Ankle Clinics 14.1 (2009): 91-104.

6. Pagenstert GI., et al. "Medial sesamoid nonunion combined with hallux valgus in athletes: a report of two cases". Foot and Ankle International 27.2 (2006):135-140.

7. Singh R and Slater N. "Hallucal sesamoid bone stress fracture; 21st century "club foot". Emergency Medicine Journal 18.4 (2001): 318.

8. Wall J and Feller JF. "Imaging of stress fractures in runners". Clinics in Sports Medicine 25.4 (2006): 781-802.

9. Biedert R and Hintermann B. "Stress Fractures of the Medial Great Toe Sesamoids in Athletes". Foot and Ankle International 24.2 (2003):137-141.

10. Bichara DA., et al. "Sesamoidectomy for hallux sesamoid fractures". Foot and Ankle International 33.9 (2012): 704-706.

11. Welck MJ., et al. "Stress fractures of the foot and ankle". Injury 48.8 (2017): 1722-1726.

\section{Assets from publication with us}

- Prompt Acknowledgement after receiving the article

- Thorough Double blinded peer review

- Rapid Publication

- Issue of Publication Certificate

- High visibility of your Published work

Website: https://www.actascientific.com/

Submit Article: https://www.actascientific.com/submission.php Email us: editor@actascientific.com

Contact us: +919182824667 